\title{
A narrative review of the role of gastrointestinal dysbiosis in the pathogenesis of polycystic ovary syndrome
}

\author{
Jim Parker, FRANZCOG', Claire O'Brien, PhD², Jason Hawrelak, PhD ${ }^{3}$ \\ ${ }^{1}$ School of Medicine, Faculty of Science, Medicine and Health, University of Wollongong, Wollongong; ${ }^{2}$ Faculty of Science and Technology, University \\ of Canberra, Canberra; ${ }^{3}$ College of Health and Medicine, University of Tasmania, Tasmania, Australia
}

Diet-induced gastrointestinal dysbiosis has been hypothesized to play a significant role in stimulating an increase in gastrointestinal permeability and activating systemic inflammation in women with polycystic ovary syndrome (PCOS). We reviewed the current proof-of-concept studies on the proposed mechanism of dysbiosis in the pathogenesis of PCOS. A literature search was performed to identify articles on changes in the intestinal microbiome (dysbiosis) and increased intestinal mucosal permeability involving lipopolysaccharide (LPS), LPS-binding protein (LPS-BP), and zonulin. We also searched for systematic reviews and meta-analyses that synthesized the results of studies on the therapeutic effects of prebiotics, probiotics, or synbiotics in women with PCOS. Our search was confined to human studies between 2012 and 2021 using the PubMed, Scopus, and Cochrane databases. Thirty-one studies met the inclusion criteria (14 microbiota, 1 LPS, 1 LPS-BP, 1 LPS and LPS-BP, 5 zonulin, 9 systematic reviews). Our analysis revealed that most studies reported reduced alpha diversity and dysbiosis in women with PCOS. Preliminary studies suggest that LPS, LPS-BP, and zonulin may be involved in the pathophysiology of increased intestinal permeability. Treatment of PCOS with prebiotics, probiotics, and synbiotics appears to have a range of beneficial effects on metabolic and biochemical profiles. This review highlights the need for continued research into the pathophysiological mechanisms of dysbiosis and the clinical efficacy of prebiotics, probiotics, and synbiotics in women with PCOS.

Keywords: Dysbiosis; Lipopolysaccharides; Polycystic ovary syndrome; Probiotics; Zonulin

\section{Introduction}

Polycystic ovary syndrome (PCOS) is both a metabolic and endocrine syndrome that affects $6-15 \%$ of reproductive-age women, or approximately 200 million globally, and is believed to be increasing in frequency [1-4]. PCOS is now being recognized as an ecological condition that arises in genetically susceptible women due to a mismatch between ancestral inherited adaptive genetic polymorphisms and modern lifestyle [5]. This evolving ecological view of PCOS takes into account the interaction between lifestyle and environmental factors, such as diet and activity levels, with developmentally programmed metabolic and endocrine pathways [3,5-7]. This view supports the recommendations of the 2018 International Guidelines that the first-line management of women with PCOS should focus on lifestyle-based approaches [8]. The pathogenesis of many chronic diseases, particularly a range of metabolic diseases associated with PCOS, such as obesity, type-2 diabetes, metabolic syndrome, and gestational diabetes, are also being considered from an evolutionary perspective $[5,6,9]$.

The dysbiosis of gut microbiota theory proposed by Tremellen and Pearce explains the key steps in the development of

Received: 2021.06.10. Revised: 2021.08.17. Accepted: 2021.12.06. Corresponding author: Jim Parker, FRANZCOG

School of Medicine, Faculty of Science, Medicine and Health, University of Wollongong Australia, Northfields Ave, Wollongong NSW 2500, Australia

E-mail: jimparker@ozemail.com.au

https://orcid.org/0000-0002-5018-5555

Articles published in Obstet Gynecol Sci are open-access, distributed under the terms of the Creative Commons Attribution Non-Commercial License (http://creativecommons. org/licenses/by-nc/3.0/) which permits unrestricted non-commercial use, distribution, and reproduction in any medium, provided the original work is properly cited.

Copyright $\odot 2022$ Korean Society of Obstetrics and Gynecology 


\section{Obstetrics \& Gynecology Science}

Jim Parker, et al. Dysbiosis and the pathogenesis of PCOS

PCOS [10]. This theory proposes that a high-fat, high-sugar, low-fiber diet results in dysbiosis of the gastrointestinal (Gl) microbiota, thereby increasing gut permeability and translocation of endotoxins into the circulation. This subsequently leads to pro-inflammatory cytokine release and immunological and metabolic changes, including impaired insulin receptor function. Then, high serum insulin stimulates excessive testosterone production in the ovary, leading to impaired follicle development and the establishment of PCOS [10]. Proofof-concept studies have aimed to elucidate whether shifts in the microbiota of women with PCOS are associated with the pathogenesis of the disease. Studies evaluating changes in lipopolysaccharide (LPS), LPS-binding protein (LPS-BP), zOnulin, mucosal permeability, immune system activation, and reversal of dysbiosis with diet, prebiotics, probiotics, or synbiotics have been performed to further investigate the role of dysbiosis in the pathogenesis of PCOS.

The objective of this study was to review the literature related to the potential role of diet-induced gastrointestinal dysbiosis in the pathogenesis of PCOS, summarize the studies investigating specific mechanisms of increased intestinal permeability involving LPS, LPS-BP, and zonulin, and discuss the evidence related to treatment with prebiotics, probiotics, and synbiotics. The results are presented as a narrative review of the published literature.

\section{Methods}

We conducted a literature review using the PubMed, Scopus, and Cochrane databases. A combination of medical subject headings and keywords were used, including the search terms polycystic ovary syndrome, PCOS, dysbiosis, dysbiosis of gut microbiota theory, lipopolysaccharide, lipopolysaccharide-binding protein, zonulin, mucosal permeability, endotoxemia, and treatment of polycystic ovary syndrome with prebiotics, probiotics, or synbiotics. We confined our search criteria to research articles on human studies published in English between January 2012 and August 2021. The selection criteria for the narrative review included original articles (randomized and non-randomized controlled trials, prospective observational studies, retrospective cohort studies, and case-control studies) and systematic reviews.

Studies were included if they reported outcomes related to gastrointestinal microbiome and intestinal permeability, LPS,
LPS-BP, zonulin, endotoxemia, and treatment responses following prebiotics, probiotics, or synbiotics in human subjects. Articles were excluded if they were not in English, were performed using animal models, or investigated outcomes related to oral or vaginal microbiomes. This review was conducted according to the Preferred Reporting Items for Systematic Reviews and Meta-Analyses (PRISMA) statement guidelines. The results are presented as a narrative review of the available evidence examining the role of dysbiosis, increased intestinal mucosal permeability, and treatment of women with PCOS with prebiotics, probiotics, and synbiotics. No attempt was made to combine the results into a formal systematic review or meta-analysis due to the variety of different subject areas reviewed and the degree of heterogeneity in studies reporting similar components of the pathophysiology of the disease.

\section{Results}

The initial literature review identified 683 records from the PubMed, Scopus, and Cochrane databases. Thirty-one duplicates were removed, and 552 reports were assessed by screening titles and abstracts. Forty full text articles were reviewed, while nine articles were excluded since they did not meet the inclusion criteria. In all, we identified 31 studies that met the inclusion criteria. Details of the selection process are shown in the PRISMA flow diagram (Fig. 1).

We have summarized the relevant studies and reported the findings in the 9 sections: 1 . Role of dysbiosis of gut microbiota in the pathogenesis of PCOS; 2 . Dysbiosis; 3. Lipopolysaccharide; 4. Lipopolysaccharide-binding protein; 5. Zonulin treatment and increased mucosal permeability; 6 . Role of prebiotics, probiotics, and synbiotics in the treatment of women with PCOS; 7. Prebiotics; 8. Probiotics; and 9. Synbiotics.

\section{The role of dysbiosis of gut microbiota in the pathogenesis of PCOS}

The human gut microbiota comprises viruses, fungi, parasites, archaea, and bacteria that have adapted to live on the mucosal surface of the intestine or in its lumen [11]. A vast majority of the literature in this field is related to the bacterial component of the microbiota in PCOS [10,12-14]. The human gut bacterial microbiota is largely acquired around 


\section{Obstetrics \& Gynecology Science}

Vol. 65 , No. 1, 2022

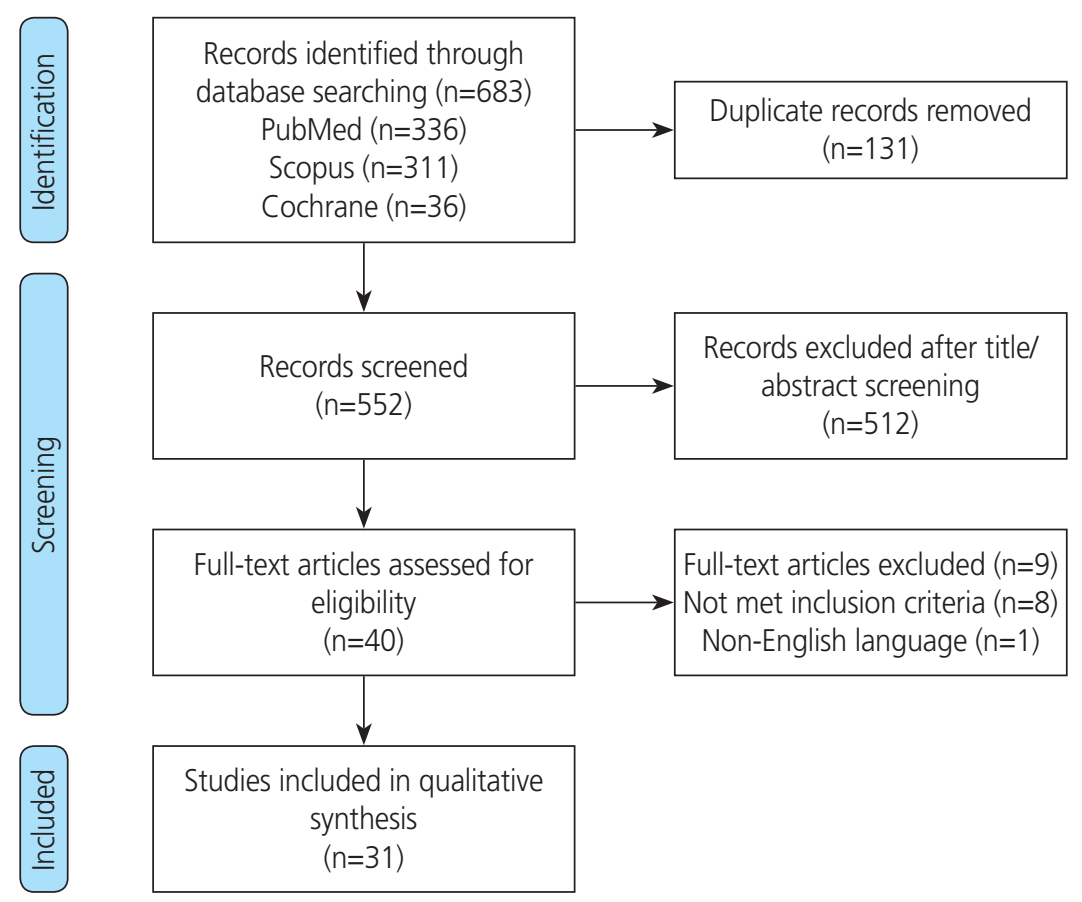

Fig. 1. PRISMA (preferred reporting items for systematic reviews and meta-analyses) flow diagram of study selection.

the time of birth and stabilizes at approximately 3 years of age [15]. The gut microbiota comprises up to 1,000 different species of bacteria, the majority of which belong to the Firmicutes and Bacteroidetes phyla. There is immense interindividual variation in the taxonomic composition of the gut microbiota, even among healthy individuals; however, the function of the microbiota is similar for all individuals [16]. Humans have evolved an intimate symbiosis with the gut microbiota, which is known to influence the immune system, inflammatory pathways, gastrointestinal epithelial barrier function, endocrine system, and host metabolism $[17,18]$. These effects on human physiology and metabolism have been found to play a potential role in multiple diseases, including PCOS $[10,12,13]$.

The gut microbiota is relatively stable; however, it may undergo changes due to lifestyle, environmental chemicals, age, antibiotic use, stress, and dietary variation $[15,19]$. It is necessary to consider antecedents, attributes, and consequences of dysbiosis in order to appreciate the impact of changes in the microbiota on human health [20]. Lifestyle and environmental factors may impair the balance of the microbiota, resulting in compositional and functional alterations $[19,21]$. This imbalance in the taxonomic composition of the gut microbiota is often referred to as dysbiosis $[20,22]$.
Studies examining the association between human disease and dysbiosis reveal wide heterogeneity in microbiota profiles, which may be due to confounding host variables, such as alcohol consumption, bowel movement quality, recent antibiotic use, and other physiological and lifestyle characteristics [23]. In addition, the gut microbiota contains numerous species of bacteria that may confer the same benefit to its host, or perform the same function as other species, so that the loss of some species or strains can be compensated for by other species that can perform the same function. This "functional redundancy" may explain why different microbiota compositions are found in individuals with the same pathological condition, such as PCOS [24].

Changes in diet can rapidly and reproducibly shift the composition and diversity of the microbiota [19]. A Western diet is associated with a depletion of bacterial taxa, resulting in reduced alpha diversity, which is a measure of species diversity or richness [25]. A reduction in alpha diversity, which is considered a feature of dysbiosis, could lead to a loss of functional redundancy, resulting in an imbalanced microbiota and pathological changes in the host [24]. Compositional changes arising from reduced alpha diversity often result in a shift toward a greater abundance of pathobionts or proinflammatory bacteria. Reduced alpha diversity is also associ- 


\section{Obstetrics \& Gynecology Science}

Jim Parker, et al. Dysbiosis and the pathogenesis of PCOS

ated with numerous diseases common in Western countries, including inflammatory bowel disease, type 2 diabetes, colorectal cancer, and obesity [26-28]. The dysbiosis of gut microbiota theory proposes that diet-induced dysbiosis triggers the development of PCOS [10].

\section{Dysbiosis}

We identified 14 human studies that compared the microbiota of patients diagnosed with PCOS with a variety of control groups [29-42]. Ten of the 14 studies reported reduced alpha diversity in the PCOS group compared to the control populations $[29-31,35-40,42]$. One study reported significant compositional changes between the PCOS and control groups before and after probiotic administration; however, it did not report alpha diversity [31]. Three studies did not find any significant differences in alpha diversity [32-34]. Two of these studies included less than 17 women with PCOS and were statistically underpowered to detect a difference [32,33]. The third study showed decreased beta diversity of PCOS microbiomes and significant differences in species abundance that were found to be related to functional alterations in bile acid metabolism [34]. Taken together, these studies suggest that compositional changes in the gastrointestinal microbiome and dysbiosis are likely to play a significant role in the pathogenesis of PCOS in some women.

Although reduced alpha diversity has been consistently observed in women with PCOS, no single bacterium or causal core change has been identified [32,36-38,42]. Some studies support an expansion of species associated with mucosal inflammation and induction of high levels of pro-inflammatory cytokines and chemokines, such as Prevotella and Escherichia coli, and other LPS-producing gram-negative bacteria [29,37]; however, other studies have identified different compositional changes $[36,42]$. The lack of standardization of host variables, such as diet, geographic variation and rates of obesity in case and control subjects, functional redundancy, or differences in microbiota assessment techniques, may account for the observed differences $[23,24,43]$.

A dysbiotic microbiota in PCOS may perpetuate and exacerbate systemic dissemination of inflammatory mediators and bacterial products such as LPS, which may in turn modulate the PCOS phenotype by inducing metabolic disturbances, leading to chronic inflammation, insulin resistance (IR), and increased androgen secretion $[10,14,37]$. No study has concurrently assessed temporal variation in the gut microbiota, body mass index (BMI), and androgen levels in obese or lean women with PCOS. Indeed, this type of study would be difficult in humans, as many of these factors are non-mutually exclusive. A recent Cochrane review revealed that lifestyle changes associated with modest weight loss were associated with lower male hormone levels and reduced hirsutism, suggesting that weight loss may significantly affect factors contributing to the development of PCOS [44]. Determining whether changes in the microbiota are a cause or effect of obesity, androgen levels, or PCOS is not clinically important if a change in diet and lifestyle results in improvement in all these factors.

Zhao et al. [13] recently reviewed the role of gut microbiota in the pathogenesis of PCOS. They identified several other possible mechanisms in which the gut microbiota may be involved in the pathogenesis of PCOS, in addition to dysbiosisrelated increased gut mucosal permeability. These include processes involving increased energy absorption, multiple possible effects related to alterations of short-chain fatty acid metabolism, changes in bile acid metabolism that affect glucose and lipid metabolism and inflammation, multiple physiological effects of altered choline metabolism pathways, and modulation of gastrointestinal hormones involved in the gut-brain interaction [13]. They suggested that a greater understanding of the diverse microbial metabolic pathways involved in the pathogenesis of PCOS may open the way for more targeted treatments with prebiotics, probiotics, traditional Chinese medicine, and fecal microbiota transplantation.

Rizk et al. [43] reviewed a range of metabolites associated with gastrointestinal dysbiosis, including host-produced metabolites (lactate, trimethylamine $\mathrm{N}$-oxide, and primary bile acids), microbiota-related metabolites (short-chain fatty acids and secondary bile acids), and targeted metabolomic studies. They concluded that our current understanding is limited by significant methodological difficulties, lack of fecal metabolomic studies, and correlational studies between gut metabolites and specific microbial species and strains [43]. Taken together, this preliminary research on the gut microbiota supports the role of dysbiosis in the pathogenesis of PCOS and suggests that there may be multiple mechanistic pathways that are possible, depending on the specific genetic, dietary, environmental, and microbiota characteristics of each individual. When considered within the broader definition of dysbiosis, which includes functional alterations to host 


\section{Obstetrics \& Gynecology Science}

Vol. 65 , No. 1, 2022

physiology and metabolism [18], in addition to imbalances in the taxonomic composition of the microbiota, it is likely that dysbiosis plays a significant role in the pathogenesis of PCOS in many women with this syndrome.

Dysbiosis of gut microbiota theory proposes two key pathophysiological conditions [10]. First, a high-fat, high-glycemic low-fiber diet results in dysbiosis and release of LPS from the cell wall of gram-negative bacteria, which can traverse the intestinal wall and act as an immunostimulant. Second, dietinduced dysbiosis causes increased mucosal permeability, facilitating the transfer of LPS from the bowel lumen to the circulation, initiating inflammation secondary to metabolic endotoxemia. Several studies have investigated these pathophysiological links in women with PCOS by investigating LPS, LPS-BP, and serum zonulin levels [45-49]. Dysbiosis theory also suggests that treatment with prebiotics, probiotics, and synbiotics may help restore eubiosis, reverse pathophysiological changes, and improve the biochemical and clinical features of PCOS.

\section{Lipopolysaccharide}

González et al. [45] performed a cross-sectional study to investigate the impact of a single episode of high saturated fat ingestion on circulating LPS, tumor necrosis factor alpha (TNFa), mononuclear cell (MNC) toll-like receptor-4 (TLR4), and suppressor of cytokine signaling-3 (SOCS-3] in a group of obese and lean women with PCOS compared with matched control groups. All women had similar baseline LPS levels. The data showed increases in LPS and TLR-4 related inflammation in obese patients, with the highest increases in obese women with PCOS. Lean women had no increase in LPS levels, regardless of whether they had PCOS. The investigators commented that the observed increases in LPS and TLR-4 inflammation may be an obesity-related phenomenon that is worsened by PCOS [45]. This finding may also reflect the fact that obese women with PCOS and obese women without PCOS have a similar dysbiosis-related response to a high-fat diet challenge. Both obese and lean women with PCOS and obese women without PCOS had lipid-induced increases in TNF $\alpha$ and SOC-3 gene expression compared with lean controls. The investigators concluded that lipid-induced inflammation may be a potential mechanism of IR in PCOS independent of obesity [45].

A cross-sectional study of 144 women found elevated markers of endotoxemia in women with PCOS compared with healthy ovulatory women. Women with PCOS had significantly higher mean LPS ( $P=0.045)$, LPS to high-density lipoprotein ratio $(P=0.007)$, and LPS-BP $(P=0.01)$ [50]. All measures of endotoxemia correlated independently and positively with the inflammatory markers and ovarian volume. Taken together, the findings of these two studies suggest that women with PCOS may exhibit a pro-inflammatory state related to elevated markers of endotoxemia, including LPS and LPS-BP $[45,50]$.

\section{Lipopolysaccharide-binding protein}

Zhu et al. [46] performed a large cross-sectional study of 238 patients, investigating fasting LPS-BP levels in a mixed group of lean and obese women with PCOS compared with age-matched controls. They reported significantly increased levels of LPS-BP in both lean and obese patients with PCOS compared to age-matched controls. LPS-BP is synthesized by hepatocytes and intestinal epithelial cells in response to inflammatory cytokines [47]. LPS-BP has a dual concentration-dependent role in monocyte-mediated inflammatory responses. At low concentrations, LPS-BP enhances LPSinduced activation of MNCs. In contrast, high levels of LPS and other endotoxins stimulate an acute-phase rise in LPS$\mathrm{BP}$, which inhibits the LPS-induced inflammatory response of monocytes [47]. This appears to be a protective mechanism to prevent an overwhelming inflammatory response to high levels of bacterial endotoxins. In addition, Zhu et al. [46] reported increased levels of IR associated with elevated levels of LPS-BP in women with PCOS. The investigators proposed that endotoxemia stimulated an increase in LPS-BP, and subsequent activation of monocyte-induced inflammation may stimulate hyperinsulinemia. The combined findings of González et al. [45] and Zhu et al. [46] support the dysbiosis hypothesis that endotoxemia may result in increased LPS and LPS-BP, which bind with TLR-4 on monocytes and stimulate systemic inflammation and hyperinsulinemia. Although these initial studies support the involvement of dysbiosis in the pathogenesis of PCOS, further studies are required to investigate the significance of this mechanism.

\section{Zonulin and increased mucosal permeability}

Zonulin was initially discovered in 2000 as an endogenous human analog to the Vibrio cholerae-derived zonula occludens toxin (ZOT), which was found to induce an enterotoxic increase in intestinal permeability in rabbit and non- 


\section{Obstetrics \& Gynecology Science}

Jim Parker, et al. Dysbiosis and the pathogenesis of PCOS

human primate intestinal epithelia [51,52]. Zonulin is a prehaptoglobin protein that regulates small intestinal permeability through its effects on tight junctions (TJ), which are located between gastrointestinal epithelial cells [53]. Small intestinal bacterial membrane-derived LPS, serum inflammatory mediators, and immune cells can stimulate zonulin release [54]. Elevated serum zonulin levels have also been found to correlate with increased intestinal permeability, as demonstrated by the lactulose-mannitol (LA/MA) test [55]. Serum zonulin has subsequently been used as a biomarker for intestinal permeability using a variety of commercially available assay kits.

Zonulin has been extensively studied in celiac and autoimmune diseases [56]. More recent studies show that elevated zonulin levels and presumed altered intestinal permeability have also been observed in a range of other conditions, including obesity, type 2 diabetes, and NAFLD, which are all associated with PCOS [57-59]. A number of studies have investigated the role of zonulin in PCOS using a variety of commercially available ELISA kits [36,56-58]. The following discussion summarizes the results of the identified studies and shows the zonulin assays that are used in parentheses.

A case-control study of 78 women with PCOS and 63 BMIand age-matched controls found significantly higher serum zonulin levels in women with PCOS ( $P=0.022)$ (ELISA Kit, Immundiagnostik AG, Bensheim, Germany) [48]. Zonulin levels were also significantly correlated with homeostatic model assessment for IR (HOMA-IR), insulin sensitivity index, and more severe menstrual disorders. A second case-control study of 90 women with PCOS and $45 \mathrm{BMI}$ and age-matched controls found significantly higher zonulin levels in the PCOS group than in controls $(P<0.01)$ (ELISA-unspecified) [49]. Zonulin levels were also positively correlated with HOMA-IR in women with PCOS [49].

Lingaiah et al. [60] reported serum zonulin levels in 104 women from Finland diagnosed with PCOS as part of a longitudinal birth cohort study, compared with 203 BMI-matched non-PCOS controls. All women were aged 46 years at the time of the study. Approximately two-thirds of the study group was diagnosed by questionnaire at the age of 31 years based on a history of oligomenorrhea and hirsutism and one-third at the age of 46 years were diagnosed with PCOS based on a self-report. The investigators reported comparable serum levels of zonulin in both the study and control populations (128.0 \pm 17.0 vs. $130.9 \pm 14.0 \mathrm{ng} / \mathrm{mL}, P=0.13$ ) (ELISA
Kit, Immundiagnostik AG). Serum zonulin levels were correlated with $\mathrm{BMI}, \mathrm{IR}$, and inflammatory markers [60]. The generalizability of the results of this study is limited by the methodology and similarity of the study and control groups. All patients were aged 46 years and were diagnosed using a questionnaire based on menstrual symptoms and hirsutism without biochemical or ultrasound assessment, and there was no contemporaneous assessment of the microbiome for the presence of dysbiosis. The study group may have been more likely to have mild PCOS and, therefore, were more similar to the control group. Metabolic profiles were comparable in the study and control groups, which may reflect increased age of subjects and the possibility that women may present more similarities with advancing age regarding metabolism.

A small pilot study of 25 women with PCOS and 19 controls reported significantly increased serum zonulin levels in women with PCOS compared to controls $(P=0.006)$ (ELIZAunspecified) [36]. The significance of these findings is limited by the small sample size of the study and the inclusion of $42 \%$ of participants with mild PCOS who did not have hyperandrogenemia [36]. Another study did not find a significant difference in serum zonulin levels between women with PCOS and controls [61]. Zonulin levels were found to be low in both groups (PCOS=43.5 ng/mL and control $=42.9 \mathrm{ng} / \mathrm{mL}$, $P=0.893)$ (Elabscience Biotechnology Co., Texas, USA) [61]. This study included 45 women with PCOS and 17 controls and was terminated early due to closure of the recruitment hospital. The diagnostic criteria for PCOS were not reported, and the BMI of the PCOS and control groups were both within the normal range $\left(\mathrm{BMl}<25 \mathrm{~kg} / \mathrm{m}^{2}\right)$. In addition, there were no significant differences in metabolic parameters between subjects and controls (low-density lipoprotein, highdensity lipoprotein, triglycerides, C-reactive protein, HOMAIR) [61].

Although the combined results of these initial studies support the hypothesis that some women with PCOS have elevated zonulin levels, studies in selected subsets have reported conflicting results. These studies highlight the need for further rigorous investigation of the role of zonulin and increased intestinal permeability in women with PCOS.

Studies investigating the validity of the relationship between elevated serum zonulin and increased intestinal permeability have been questioned as a result of recently identified methodological inconsistencies in the results obtained from 


\section{Obstetrics \& Gynecology Science}

Vol. 65 , No. 1, 2022

some of the commercially available testing assays [62-64]. Ajamian et al. [62] performed a comparative study of two assays with recombinant zonulin protein using immunoprecipitation, mass spectrometry, and gel electrophoresis. The findings indicate that commercial zonulin assays did not detect the actual zonulin protein prehaptoglobin-2. Comparative assays suggested that complement C3 and haptoglobin were the likely proteins detected by the assay kits examined [62]. Although recent comparative studies have increased concerns about the validity of the currently available test kits, there is disagreement regarding the identity of the alternative proteins that are being detected by individual assays [62-64]. Scheffler et al. [63] suggested that some commercial kits identified the same target proteins and Ajamian et al. [62] found that these same assays identified different proteins.

Both complement-associated and haptoglobin proteins share similar homology and may also modulate zonulin production and intestinal barrier integrity $[53,62,65,66]$. Although the commercial kits that were examined may not help detect the actual zonulin protein, they may still contribute to obtaining results that reflect associated molecular biomarkers of intestinal permeability $[53,65,66]$. Nevertheless, the validity and predictive value of current assays require clarification. These methodological inconsistencies also raise concerns regarding the use of zonulin testing in clinical practice. More specific ELISA detection kits should be developed using reliable monoclonal antibodies against recombinant zonulin/prehaptoglobin-2 [62]. In the meantime, the more invasive LA/MA test should be used in experimental studies to investigate intestinal barrier integrity. The effects of dysbiosis could also be investigated using LPS, LPS-BP, inflammatory mediators, and measures of immune function as surrogate biomarkers for intestinal barrier dysfunction.

\section{Role of prebiotics, probiotics, and synbiotics in the treatment of women with PCOS}

Following the proposal that dysbiosis may be a contributor to the pathogenesis of PCOS, it has been hypothesized that modification of the balance of the gastrointestinal microbiota has the potential to be an effective treatment. This has resulted in a large number of studies investigating the therapeutic effects of prebiotics, probiotics, and synbiotics. We identified nine systematic reviews and meta-analyses that have synthesized results from 17 randomized controlled trials (RCTs) published between 2017 and 2021 [67-75]. The focus of individual systematic reviews has varied and has included outcomes related to anthropomorphic, biochemical, hormonal, inflammatory indices, oxidative stress, and IR. It is not possible to systematically synthesize these reviews due to the heterogeneity of the study aims and reported outcomes. The following sections provide a narrative review of the conclusions of these studies. More detailed results can be obtained from the individual reports.

\section{Prebiotics}

Prebiotics are substances that are selectively utilized by host microorganisms, conferring health benefits to the host [76]. Substances that are widely considered to meet this definition include inulin-type fructans, galactooligosaccharides, and lactulose [77]. All three of these compounds have demonstrated the capacity to significantly shift the GI microbial ecosystem in beneficial ways [78-90]. Although we were unable to identify specific publications on any of these three prebiotics individually in women with PCOS, other evidence suggests that they could be useful therapeutic options.

Inulin-type fructans have been found to decrease endotoxin absorption [81], enhance intestinal integrity, and improve both blood glucose regulation and fasting insulin in patients with type 2 diabetes and prediabetes [82]. Galactooligosaccharides have been found to improve metabolic markers (e.g., reductions in plasma insulin and C-reactive protein [CRP] levels) in patients with metabolic syndrome [83]. Lactulose has been found to decrease endotoxin absorption $[84,85]$ and improve intestinal hyperpermeability, as well as blood glucose levels and insulin response in type 2 diabetics [86]. More research is needed to evaluate the impact of these prebiotics in PCOS, as the only research to date has been limited to examining prebiotics as components of synbiotic preparations (discussed below), rather than prebiotics in isolation.

Resistant starch (RS) refers to the portion of starch and starch products in foods and food products that resist digestion in the upper gastrointestinal tract [87]. These starches share some characteristics with the prebiotics discussed above, such as indigestibility and a selective impact on the $\mathrm{Gl}$ ecosystem. Resistant starches have been found to increase the concentrations of butyrate-producing bacteria and bifidobacteria, which result in beneficial shifts in the GI microbiota $[88,89]$. Studies have also found a consistent beneficial impact of RS on glycemic control and systemic inflammation, both of which are important components in the 


\section{Obstetrics \& Gynecology Science}

Jim Parker, et al. Dysbiosis and the pathogenesis of PCOS

pathophysiology of PCOS [90]. A recent RCT compared 20 $g$ per day of RS in women with PCOS to placebo [91]. After 3 months, there were significant improvements in serum triglycerides $(P=0.001)$, total cholesterol $(P<0.001)$, low-density lipoprotein cholesterol $(P<0.001)$, hsCRP $(P=0.004)$, free testosterone $(P=0.01)$, menstrual cycle regularity $(P<0.001)$, and hirsuitism $(P<0.001)$ in women treated with RS [91]. Another publication based on this same trial reported significant reductions in waist circumference, hip circumference, weight, and $\mathrm{BMI}$ in the treatment group (all $P<0$.001) [92]. Unfortunately, this study did not evaluate the impact of RS intervention on the GI microbiota composition; therefore, the impact of RS on the ecosystem itself in women with PCOS is currently unknown.

\section{Probiotics}

Probiotics are defined as live microbes that, when ingested in adequate amounts, confer health benefits [93]. To date, most of the probiotics that have been studied originate from the genera Lactobacillus and Bifidobacterium. However, a shift has recently been noted [94], in which a range of probiotic preparations and strains have been evaluated in the treatment of PCOS, with a number of recent meta-analyses published in this area showing consistent positive results on a range of PCOS-related parameters [67-70].

In one of the most rigorous and comprehensive metaanalyses, Tabrizi et al. [71] reviewed RCTs of probiotics in women with PCOS to determine their effectiveness on clinical symptoms, glycemic control, weight loss, hormonal and lipid profiles, and markers of inflammation. A wide range of probiotic preparations and dosages were used in the 11 RCTs included in the review. Probiotic supplementation significantly decreased body weight $(P=0.01), \mathrm{BMI}(P<0.02)$, fasting plasma glucose $(P<0.001)$, HOMA-IR $(P<0.001)$, insulin levels $(P<0.001)$, hirsutism $(P<0.001)$, total testosterone levels $(P<0.001)$, VLDL cholesterol $(P<0.001)$, triglycerides $(P<0.001)$, and $C$-reactive protein $(P<0.001)$ [71]. Quantitative insulin sensitivity check index $(P<0.01)$, total antioxidant capacity $(P<0.001)$, glutathione levels $(P=0.04)$, and SHBG concentrations $(P=0.01)$ were all found to significantly increase following probiotic supplementation [71]. These data also support the role of dysbiosis in the pathogenesis of PCOS.

There are numerous potential mechanisms of action that may explain the observed beneficial effects of probiotics in women with PCOS. These include antioxidant [95], antiinflammatory [96], gastrointestinal healing [97], anti-LPS [98], sex hormone-altering [31], and metabolism-regulating actions [99], in addition to the ability of some probiotic strains to significantly impact microbiota composition $[32,100,101]$. The actions and characteristics of probiotics are strain-specific. The efficacy and mechanisms of action of one probiotic strain, or a combination of strains, cannot be extrapolated to other strains [102]. Taken together, these data suggest that the use of probiotics may be a useful therapeutic adjunct to dietary interventions, as well as other lifestyle interventions, in women with PCOS. Further studies are needed to elucidate strain-specific therapeutic mechanisms of action and to delineate which strains do not confer treatment benefits.

\section{Synbiotics}

Synbiotics are mixtures of live microorganisms and substrates selectively utilized by host microbes; when consumed, they confer a health benefit to the host [103]. Generally, they contain both probiotic and prebiotic components. Synbiotics can be composed of a wide range of probiotic strains in combination with a variety of prebiotic and prebiotic dosages. As a class of agents, synbiotics have a growing evidence base for the treatment of obesity [104], gestational diabetes [105], type 1 [106], and type 2 diabetes [107]. It is not surprising that synbiotics have also been evaluated in the treatment of women with PCOS, since PCOS is part of this related cluster of metabolic conditions [5,108-110].

A recent $R C T$ investigated the impact of synbiotic preparation on sex hormone profile and glycemic and anthropometric indices in subjects with PCOS. After 8 weeks, subjects who received the synbiotic preparation (Lactobacillus rhamnosus GG in combination with unspecified strains of Bacillus coagulans and Bacillus indicus, as well as the prebiotic inulin) had significant reductions in HOMA-IR, body weight, BMI, waist circumference, fasting blood sugar, insulin, and serum testosterone compared to the placebo group (all $P<0.05$ ) [111]. Darvishi et al. [112] evaluated the efficacy of different synbiotic preparations (containing fructooligosaccharides as the prebiotic and unspecified strains of Lactobacillus casei, Lactobacillus rhamnosus, Lactobacillus bulgaricus, Lactobacillus acidophilus, Bifidobacterium longum, and Streptococcus thermophilus) in the treatment of PCOS. After an 8-week administration period, several metabolic markers were significantly improved, including serum fasting glucose $(P=0.02)$, 


\section{Obstetrics \& Gynecology Science}

Vol. 65, No. 1, 2022

HOMA-IR ( $P=0.001)$, HDL-cholesterol $(P=0.02)$, BMI $(P=0.02)$, body weight $(P=0.02)$, and waist circumference $(P=0.01)$, compared to placebo [112].

Given the uniqueness of different synbiotic preparations, which may contain a variety of probiotic strains and different prebiotic compounds, each unique synbiotic preparation needs to be individually evaluated. This is necessary to ascertain both their effectiveness in managing PCOS and their specific mechanisms of action, since it is possible that different synbiotics will act via different mechanisms and that not all synbiotics will be effective [113]. However, research to date suggests that synbiotics are a promising class of agents for the treatment of PCOS. This may be due to several different mechanisms, including microbiota modification, enhanced gastrointestinal integrity, and anti-LPS actions, as previously discussed in the prebiotic and probiotic sections.

\section{Conclusion}

Overall, the available research supports the hypothesis that diet-induced dysbiosis of the gastrointestinal microbiota is likely to play a role in the pathogenesis of PCOS. Several lines of emerging evidence suggest that dysbiosis may result in increased gastrointestinal permeability and the development of the observed metabolic, endocrine, and phenotypic features of PCOS. Multiple mechanisms may be involved, in addition to dysbiosis-related permeability changes and LPS-induced systemic inflammation. These include diet-induced microbial metabolic and signaling mechanisms involving luminal nutrient sensing pathways and metabolism of bile acids, hormones, choline, and short-chain fatty acids. These potential mechanisms have not been reviewed in the present study.

It seems likely that a combination of diet and other lifestyle-related factors may be associated with the pathophysiological features of PCOS depending on the composition of the microbiota, microbial metabolism, and underlying genetic predispositions. PCOS represents an opportunity for the early diagnosis of metabolic issues that are predictive of significant future morbidity and mortality. Therefore, the precise details of the wide range of pathogenic mechanisms that may be involved should be the focus of future research on this relevant and common disease. Nevertheless, it is noteworthy that the metabolic, reproductive, and phenotypic features of PCOS are modifiable and reversible in most women with PCOS by implementing lifestyle-based interventions, regardless of the pathophysiological mechanisms involved.

\section{Conflict of interest}

No potential conflict of interest relevant to this article.

\section{Ethical approval}

This study did not require approval from the Institutional Review Board because no patient data were included in this study.

\section{Patient consent}

Written informed consent and the use of images from patients were not required for publication.

\section{Funding information}

None.

\section{References}

1. Ding T, Hardiman PJ, Petersen I, Wang FF, Qu F, Baio G. The prevalence of polycystic ovary syndrome in reproductive-aged women of different ethnicity: a systematic review and meta-analysis. Oncotarget 2017;8:96351-8.

2. Boyle JA, Cunningham J, O'Dea K, Dunbar T, Norman RJ. Prevalence of polycystic ovary syndrome in a sample of Indigenous women in Darwin, Australia. Med J Aust 2012; 196:62-6.

3. Pathak G, Nichter M. Polycystic ovary syndrome in globalizing India: an ecosocial perspective on an emerging lifestyle disease. Soc Sci Med 2015;146:21-8.

4. M. Szmigiera. Proportion of selected age groups of world population in 2021, by region [Internet]. Hamburg (DEU): Statista; c2021 [cited 2021 May 1]. Available form: https://www.statista.com/statistics/265759/worldpopulation-by-age-and-region/. 


\section{Obstetrics \& Gynecology Science}

Jim Parker, et al. Dysbiosis and the pathogenesis of PCOS

5. Parker J. Understanding the pathogenesis of polycystic ovary syndrome: a transgenerational evolutionary adaptation to lifestyle and the environment. J ACNEM 2020;39:18-26.

6. Charifson MA, Trumble BC. Evolutionary origins of polycystic ovary syndrome: an environmental mismatch disorder. Evol Med Public Health 2019;2019:50-63.

7. Azziz R, Dumesic DA, Goodarzi MO. Polycystic ovary syndrome: an ancient disorder? Fertil Steril 2011;95:1544-8.

8. Teede H, Misso M, Costello M, Dokras A, Laven J, Moran $\mathrm{L}$, et al. International evidence-based guideline for the assessment and management of polycystic ovary syndrome 2018 [Internet]. NHMRC Canberra (ACT); c2018 [cited 2020 Out 15]. Available form: https:// www.monash.edu/_data/assets/pdf_file/0004/ 1412644/PCOS_Evidence-Based-Guidelines_ 20181009.pdf.

9. Benton ML, Abraham A, LaBella AL, Abbot P, Rokas A, Capra JA. The influence of evolutionary history on human health and disease. Nat Rev Genet 2021;22:26983.

10. Tremellen K, Pearce K. Dysbiosis of gut microbiota (DOGMA)--a novel theory for the development of polycystic ovarian syndrome. Med Hypotheses 2012;79: 104-12.

11. Nuriel-Ohayon M, Neuman $H$, Koren O. Microbial changes during pregnancy, birth, and infancy. Front Microbiol 2016;7:1031.

12. Yurtdaş G, Akdevelioğlu Y. A new approach to polycystic ovary syndrome: the gut microbiota. J Am Coll Nutr 2020;39:371-82.

13. Zhao $X$, Jiang $Y$, Xi $H$, Chen $L$, Feng $X$. Exploration of the relationship between gut microbiota and polycystic ovary syndrome (PCOS): a review. Geburtshilfe Frauenheilkd 2020;80:161-71.

14. He FF, Li YM. Role of gut microbiota in the development of insulin resistance and the mechanism underlying polycystic ovary syndrome: a review. J Ovarian Res 2020;13:73.

15. Yatsunenko T, Rey FE, Manary MJ, Trehan I, Dominguez-Bello MG, Contreras M, et al. Human gut microbiome viewed across age and geography. Nature 2012;486:222-7.

16. Human Microbiome Project Consortium. Structure, function and diversity of the healthy human microbiome. Nature 2012;486:207-14.

17. Tuddenham $S$, Sears $C L$. The intestinal microbiome and health. Curr Opin Infect Dis 2015;28:464.

18. García-Montero C, Fraile-Martínez O, Gómez-Lahoz AM, Pekarek L, Castellanos AJ, Noguerales-Fraguas F, et al. Nutritional components in western diet versus mediterranean diet at the gut microbiota-immune system interplay. Implications for health and disease. Nutrients 2021;13:699.

19. David LA, Maurice CF, Carmody RN, Gootenberg $\mathrm{DB}$, Button JE, Wolfe $\mathrm{BE}$, et al. Diet rapidly and reproducibly alters the human gut microbiome. Nature 2014;505:559-63.

20. Perez NB, Dorsen C, Squires A. Dysbiosis of the gut microbiome: a concept analysis. J Holist Nurs 2020;38:223-32.

21. Moschen AR, Wieser $V$, Tilg $H$. Dietary factors: major regulators of the gut's microbiota. Gut Liver 2012;6:411-6.

22. Hawrelak JA, Myers SP. The causes of intestinal dysbiosis: a review. Altern Med Rev 2004;9:180-97.

23. Vujkovic-Cvijin I, Sklar J, Jiang L, Natarajan L, Knight $\mathrm{R}$, Belkaid Y. Host variables confound gut microbiota studies of human disease. Nature 2020;587:448-54.

24. Moya A, Ferrer M. Functional redundancy-induced stability of gut microbiota subjected to disturbance. Trends Microbiol 2016;24:402-13.

25. Partula V, Mondot S, Torres MJ, Kesse-Guyot E, Deschasaux M, Assmann K, et al. Associations between usual diet and gut microbiota composition: results from the Milieu Intérieur cross-sectional study. Am J Clin Nutr 2019;109:1472-83.

26. Manichanh C, Rigottier-Gois L, Bonnaud E, Gloux K, Pelletier $E$, Frangeul $L$, et al. Reduced diversity of faecal microbiota in Crohn's disease revealed by a metagenomic approach. Gut 2006;55:205-11.

27. O'Brien CL, Pavli P, Gordon DM, Allison GE. Detection of bacterial DNA in lymph nodes of Crohn's disease patients using high throughput sequencing. Gut 2014;63:1596-606.

28. Wang T, Cai G, Qiu Y, Fei N, Zhang M, Pang X, et al. Structural segregation of gut microbiota between colorectal cancer patients and healthy volunteers. ISME J 2012;6:320-9. 


\section{Obstetrics \& Gynecology Science}

Vol. 65, No. 1, 2022

29. Mammadova G, Ozkul C, Yilmaz Isikhan S, Acikgoz A, Yildiz BO. Characterization of gut microbiota in polycystic ovary syndrome: findings from a lean population. Eur J Clin Invest 2021;51:e13417.

30. Haudum C, Lindheim L, Ascani A, Trummer C, Horvath A, Münzker J, et al. Impact of short-term isoflavone intervention in polycystic ovary syndrome (PCOS) patients on microbiota composition and metagenomics. Nutrients 2020;12:1622.

31. Zhang J, Sun Z, Jiang S, Bai X, Ma C, Peng Q, et al. Probiotic bifidobacterium lactis $\vee 9$ regulates the secretion of sex hormones in polycystic ovary syndrome patients through the gut-brain axis. mSystems 2019;4:e00017-19.

32. Insenser M, Murri M, Del Campo R, Martínez-García MÁ, Fernández-Durán E, Escobar-Morreale HF. Gut microbiota and the polycystic ovary syndrome: influence of sex, sex hormones, and obesity. J Clin Endocrinol Metab 2018;103:2552-62.

33. Eyupoglu ND, Ergunay K, Acikgoz A, Akyon Y, Yilmaz E, Yildiz BO. Gut Microbiota and oral contraceptive use in overweight and obese patients with polycystic ovary syndrome. J Clin Endocrinol Metab 2020;105:dgaa600.

34. Qi X, Yun C, Sun L, Xia J, Wu Q, Wang Y, et al. Gut microbiota-bile acid-interleukin-22 axis orchestrates polycystic ovary syndrome. Nat Med 2019;25:122533.

35. Jobira B, Frank DN, Pyle L, Silveira LJ, Kelsey MM, Garcia-Reyes $Y$, et al. Obese adolescents with PCOS have altered biodiversity and relative abundance in gastrointestinal microbiota. J Clin Endocrinol Metab 2020;105:e2134-44.

36. Lindheim L, Bashir M, Münzker J, Trummer C, Zachhuber $\mathrm{V}$, Leber $\mathrm{B}$, et al. Alterations in gut microbiome composition and barrier function are associated with reproductive and metabolic defects in women with polycystic ovary syndrome (PCOS): a pilot study. PLOS One 2017;12:e0168390.

37. Liu R, Zhang $C$, Shi $Y$, Zhang F, Li L, Wang $X$, et al. Dysbiosis of gut microbiota associated with clinical parameters in polycystic ovary syndrome. Front Microbiol 2017;8:324.

38. Torres PJ, Siakowska M, Banaszewska B, Pawelczyk L, Duleba AJ, Kelley ST, et al. Gut microbial diversity in women with polycystic ovary syndrome correlates with hyperandrogenism. J Clin Endocrinol Metab 2018;103:1502-11.

39. Chen F, LAI Z, XU Z. Analysis of the gut microbial composition in polycystic ovary syndrome with acne. Zigong Matern Child Heal Hosp 2019;35:2246-51.

40. Zhou L, Ni Z, Cheng W, Yu J, Sun S, Zhai D, et al. Characteristic gut microbiota and predicted metabolic functions in women with PCOS. Endocr Connect 2020;9:63-73.

41. Garcia-Beltran C, Malpique R, Carbonetto B, González-Torres P, Henares D, Brotons P, et al. Gut microbiota in adolescent girls with polycystic ovary syndrome: effects of randomized treatments. Pediatr Obes 2021;16:e12734.

42. Zeng B, Lai Z, Sun L, Zhang Z, Yang J, Li Z, et al. Structural and functional profiles of the gut microbial community in polycystic ovary syndrome with insulin resistance (IR-PCOS): a pilot study. Res Microbiol 2019;170:43-52.

43. Rizk MG, Thackray VG. Intersection of polycystic ovary syndrome and the gut microbiome. J Endocr Soc 2020;5:bvaa177.

44. Lim SS, Hutchison SK, Van Ryswyk E, Norman RJ, Teede HJ, Moran LJ. Lifestyle changes in women with polycystic ovary syndrome. Cochrane Database Syst Rev 2019;3:CD007506.

45. González F, Considine RV, Abdelhadi OA, Acton AJ. Saturated fat ingestion promotes lipopolysaccharidemediated inflammation and insulin resistance in polycystic ovary syndrome. J Clin Endocrinol Metab 2019;104:934-46.

46. Zhu Q, Zhou H, Zhang A, Gao R, Yang S, Zhao C, et al. Serum LBP Is associated with insulin resistance in women with PCOS. PLoS One 2016;11:e0145337.

47. Gutsmann T, Müller M, Carroll SF, MacKenzie RC, Wiese A, Seydel U. Dual role of lipopolysaccharide (LPS)binding protein in neutralization of LPS and enhancement of LPS-induced activation of mononuclear cells. Infect Immun 2001;69:6942-50.

48. Zhang D, Zhang L, Yue F, Zheng Y, Russell R. Serum zonulin is elevated in women with polycystic ovary syndrome and correlates with insulin resistance and severity of anovulation. Eur J Endocrinol 2015;172:2936.

49. Anik Ilhan G, Yildizhan B. Evaluation of zonulin, 


\section{Obstetrics \& Gynecology Science}

Jim Parker, et al. Dysbiosis and the pathogenesis of PCOS

a marker of intestinal permeability as a novel biomarker in polycystic ovary syndrome. Fertil Steril 2018;110:e117.

50. Banaszewska B, Siakowska M, Chudzicka-Strugala I, Chang RJ, Pawelczyk L, Zwozdziak, et al. Elevation of markers of endotoxemia in women with polycystic ovary syndrome. Hum Reprod 2020;35:2303-11.

51. Fasano A, Uzzau S, Fiore C, Margaretten K. The enterotoxic effect of zonula occludens toxin on rabbit small intestine involves the paracellular pathway. Gastroenterology 1997;112:839-46.

52. Larkin M. Out with the jab, in with the painless pills. Lancet 1997;349:1676.

53. Fasano A. Zonulin and its regulation of intestinal barrier function: the biological door to inflammation, autoimmunity, and cancer. Physiol Rev 2011;91:151-75.

54. Fasano A. Intestinal permeability and its regulation by zonulin: diagnostic and therapeutic implications. Clin Gastroenterol Hepatol 2012;10:1096-100.

55. Sapone A, de Magistris L, Pietzak M, Clemente MG, Tripathi A, Cucca F, et al. Zonulin upregulation is associated with increased gut permeability in subjects with type 1 diabetes and their relatives. Diabetes 2006;55:1443-9.

56. Fasano A. Zonulin, regulation of tight junctions, and autoimmune diseases. Ann N Y Acad Sci 2012;1258: 25-33.

57. Kim AS, Ko HJ. Plasma concentrations of zonulin are elevated in obese men with fatty liver disease. Diabetes Metab Syndr Obes 2018;11:149-57.

58. Aasbrenn M, Lydersen S, Farup PG. Changes in serum zonulin in individuals with morbid obesity after weight-loss interventions: a prospective cohort study. BMC Endocr Disord 2020;20:108.

59. Yuan JH, Xie QS, Chen GC, Huang CL, Yu T, Chen QK, et al. Impaired intestinal barrier function in type 2 diabetic patients measured by serum LPS, zonulin, and IFABP. J Diabetes Complications 2021;35:107766.

60. Lingaiah S, Arffman RK, Morin-Papunen L, Tapanainen JS, Piltonen T. Markers of gastrointestinal permeability and dysbiosis in premenopausal women with PCOS: a case-control study. BMJ Open 2021;11:e045324.

61. Cetin Z, Kosem A, Can B, Baser O, Catak M, Turhan $\mathrm{T}$, et al. Serum zonulin level is not elevated in patients with polycystic ovary syndrome without metabolic syn- drome. Arch Gynecol Obstet 2019;300:1785-90.

62. Ajamian M, Steer D, Rosella G, Gibson PR. Serum zonulin as a marker of intestinal mucosal barrier function: may not be what it seems. PLoS One 2019;14:e0210728.

63. Scheffler L, Crane A, Heyne H, Tönjes A, Schleinitz D, Ihling $\mathrm{CH}$, et al. Widely used commercial ELISA for human Zonulin reacts with Complement C3 rather than pre-Haptoglobin 2 [Internet]. NY (USA): CSH; c2017 [cited 2021 Apr 17]. Available from: http://www. biorxiv.org/content/biorxiv/early/2017/06/30/157578. full.pdf.

64. Scheffler L, Crane A, Heyne H, Tönjes A, Schleinitz D, Ihling $\mathrm{CH}$, et al. Widely used commercial elisa does not detect precursor of haptoglobin2, but recognizes properdin as a potential second member of the zonulin family. Front Endocrinol (Lausanne) 2018;9:22.

65. Sina C, Kemper C, Derer S. The intestinal complement system in inflammatory bowel disease: shaping intestinal barrier function. Semin Immunol 2018;37:66-73.

66. Sünderhauf A, Skibbe K, Preisker S, Ebbert K, Verschoor A, Karsten CM, et al. Regulation of epithelial cell expressed C3 in the intestine - relevance for the pathophysiology of inflammatory bowel disease? Mol Immunol 2017;90:227-38.

67. Liao D, Zhong C, Li C, Mo L, Liu Y. Meta-analysis of the effects of probiotic supplementation on glycemia, lipidic profiles, weight loss and C-reactive protein in women with polycystic ovarian syndrome. Minerva Med 2018;109:479-87.

68. Heshmati J, Farsi F, Yosaee S, Razavi M, Rezaeinejad M, Karimie $E$, et al. The effects of probiotics or synbiotics supplementation in women with polycystic ovarian syndrome: a systematic review and meta-analysis of randomized clinical trials. Probiotics Antimicrob Proteins 2019;11:1236-47.

69. Hadi A, Moradi S, Ghavami A, Khalesi S, Kafeshani M. Effect of probiotics and synbiotics on selected anthropometric and biochemical measures in women with polycystic ovary syndrome: a systematic review and meta-analysis. Eur J Clin Nutr 202074:543-7.

70. Shamasbi SG, Ghanbari-Homayi S, Mirghafourvand $M$. The effect of probiotics, prebiotics, and synbiotics on hormonal and inflammatory indices in women with polycystic ovary syndrome: a systematic review and 


\section{Obstetrics \& Gynecology Science}

Vol. 65 , No. 1, 2022

meta-analysis. Eur J Nutr 2020;59:433-50.

71. Tabrizi R, Ostadmohammadi V, Akbari M, Lankarani KB, Vakili S, Peymani P, et al. The effects of probiotic supplementation on clinical symptom, weight loss, glycemic control, lipid and hormonal profiles, biomarkers of inflammation, and oxidative stress in women with polycystic ovary syndrome: a systematic review and meta-analysis of randomized controlled trials. Probiotics Antimicrob Proteins 2019 Jun 4 [Epub]. https://doi. ory/10.1007/s12602-019-09559-0.

72. Kazemi A, Soltani S, Ghorabi S, Keshtkar A, Daneshzad $E$, Nasri $F$, et al. Effect of probiotic and synbiotic supplementation on inflammatory markers in health and disease status: a systematic review and meta-analysis of clinical trials. Clin Nutr 2020;39:789-819.

73. Cozzolino M, Vitagliano A, Pellegrini L, Chiurazzi M, Andriasani A, Ambrosini $G$, et al. Therapy with probiotics and synbiotics for polycystic ovarian syndrome: a systematic review and meta-analysis. Eur J Nutr 2020;59:2841-56.

74. Miao C, Guo Q, Fang X, Chen Y, Zhao Y, Zhang Q. Effects of probiotic and synbiotic supplementation on insulin resistance in women with polycystic ovary syndrome: a meta-analysis. J Int Med Res 2021;49:3000605211031758.

75. Li Y, Tan Y, Xia G, Shuai J. Effects of probiotics, prebiotics, and synbiotics on polycystic ovary syndrome: a systematic review and meta-analysis. Crit Rev Food Sci Nutr. 2021 Jul 21 [Epub]. https://doi.ory/10.1080/1040 8398.2021 .1951155$.

76. Gibson GR, Hutkins R, Sanders ME, Prescott SL, Reimer RA, Salminen SJ, et al. Expert consensus document: the international scientific association for probiotics and prebiotics (ISAPP) consensus statement on the definition and scope of prebiotics. Nat Rev Gastroenterol Hepatol 2017;14:491-502.

77. Hawrelak J. Prebiotics, synbiotics and colonic foods. In: Murray M, Pizzorno J, editors. Textbook of natural medicine. 5th ed. Amsterdam: Elsevier; 2020. p.797808.

78. Gibson GR, Beatty ER, Wang X, Cummings JH. Selective stimulation of bifidobacteria in the human colon by oligofructose and inulin. Gastroenterology 1995;108:975-82.

79. Ballongue J, Schumann C, Quignon P. Effects of lactu- lose and lactitol on colonic microflora and enzymatic activity. Scand J Gastroenterol Suppl 1997;222:41-4.

80. Vulevic J, Drakoularakou A, Yaqoob P, Tzortzis G, Gibson GR. Modulation of the fecal microflora profile and immune function by a novel trans-galactooligosaccharide mixture (B-GOS) in healthy elderly volunteers. Am J Clin Nutr 2008;88:1438-46.

81. Dehghan P, Gargari BP, Jafar-Abadi MA, Aliasgharzadeh $A$. Inulin controls inflammation and metabolic endotoxemia in women with type 2 diabetes mellitus: a randomized-controlled clinical trial. Int J Food Sci Nutr 2014;65:117-23.

82. Wang L, Yang $H$, Huang $H$, Zhang C, Zuo HX, Xu P, et al. Inulin-type fructans supplementation improves glycemic control for the prediabetes and type 2 diabetes populations: results from a GRADE-assessed systematic review and dose-response meta-analysis of 33 randomized controlled trials. J Transl Med 2019;17:410.

83. Vulevic J, Juric A, Tzortzis G, Gibson GR. A mixture of trans-galactooligosaccharides reduces markers of metabolic syndrome and modulates the fecal microbiota and immune function of overweight adults. J Nutr 2013;143:324-31.

84. Pain JA, Bailey ME. Experimental and clinical study of lactulose in obstructive jaundice. Br J Surg 1986;73: 775-8.

85. Jain L, Sharma BC, Srivastava S, Puri SK, Sharma P, Sarin S. Serum endotoxin, inflammatory mediators, and magnetic resonance spectroscopy before and after treatment in patients with minimal hepatic encephalopathy. J Gastroenterol Hepatol 2013;28:1187-93.

86. Bianchi G, Ronchi M, Marchesini G. Effect of lactulose on carbohydrate metabolism and diabetes mellitus. Scand J Gastroenterol Suppl 1997;222:62-4.

87. Sofi SA, Anjum A, Awsi J. Resistant starch as functional ingredient: a review. Int J Food Sci Nutr 2017;2:195-9.

88. Venkataraman A, Sieber JR, Schmidt AW, Waldron C, Theis KR, Schmidt TM. Variable responses of human microbiomes to dietary supplementation with resistant starch. Microbiome 2016;4:33.

89. Bendiks ZA, Knudsen KEB, Keenan MJ, Marco ML. Conserved and variable responses of the gut microbiome to resistant starch type 2. Nutr Res 2020;77:1228.

90. Halajzadeh J, Milajerdi A, Reiner Ž, Amirani E, Kolah- 


\section{Obstetrics \& Gynecology Science}

Jim Parker, et al. Dysbiosis and the pathogenesis of PCOS

dooz F, Barekat M, et al. Effects of resistant starch on glycemic control, serum lipoproteins and systemic inflammation in patients with metabolic syndrome and related disorders: a systematic review and metaanalysis of randomized controlled clinical trials. Crit Rev Food Sci Nutr 2020;60:3172-84.

91. Gholizadeh Shamasbi S, Dehgan P, Mohammad-Alizadeh Charandabi S, Aliasgarzadeh A, Mirghafourvand $M$. The effect of resistant dextrin as a prebiotic on metabolic parameters and androgen level in women with polycystic ovarian syndrome: a randomized, tripleblind, controlled, clinical trial. Eur J Nutr 2019;58:62940.

92. Shamasbi SG, Dehghan P, Charandabi SMA, Aliasgarzadeh A, Mirghafourvand M. Effect of prebiotic on anthropometric indices in women with polycystic ovarian syndrome: a triple-blind, randomized, controlled clinical trial. Iran Red Crescent Med J 2018;20:e67270.

93. Hill C, Guarner F, Reid G, Gibson GR, Merenstein DJ, Pot $B$, et al. Expert consensus document. The International Scientific Association for Probiotics and Prebiotics consensus statement on the scope and appropriate use of the term probiotic. Nat Rev Gastroenterol Hepatol 2014;11:506-14.

94. Perraudeau F, McMurdie P, Bullard J, Cheng A, Cutcliffe $C$, Deo A, et al. Improvements to postprandial glucose control in subjects with type 2 diabetes: a multicenter, double blind, randomized placebo-controlled trial of a novel probiotic formulation. BMJ Open Diabetes Res Care 2020;8:e001319.

95. Mishra V, Shah C, Mokashe N, Chavan R, Yadav H, Prajapati J. Probiotics as potential antioxidants: a systematic review. J Agric Food Chem 2015;63:3615-26.

96. Imaoka A, Shima T, Kato K, Mizuno S, Uehara T, Matsumoto $S$, et al. Anti-inflammatory activity of probiotic Bifidobacterium: enhancement of IL-10 production in peripheral blood mononuclear cells from ulcerative colitis patients and inhibition of IL-8 secretion in HT-29 cells. World J Gastroenterol 2008;14:2511-6.

97. Guillot CC, Bacallao EG, Dominguez MSC, Garcia MF, Gutierrez PM. Effects of Saccharomyces boulardii in children with chronic diarrhoea, especially cases due to giardiasis. Rev Mex De Puericultura Y Pediatria 1995;2:15.

98. In Kim H, Kim JK, Kim JY, Jang SE, Han MJ, Kim DH.
Lactobacillus plantarum LC27 and Bifidobacterium longum LC67 simultaneously alleviate high-fat diet-induced colitis, endotoxemia, liver steatosis, and obesity in mice. Nutr Res 2019;67:78-89.

99. Kocsis T, Molnár B, Németh $D$, Hegyi P, Szakács $Z$, Bálint $A$, et al. Probiotics have beneficial metabolic effects in patients with type 2 diabetes mellitus: a meta-analysis of randomized clinical trials. Sci Rep 2020;10:11787.

100. del Campo R, Garriga M, Pérez-Aragón A, Guallarte $P$, Lamas $A$, Máiz $L$, et al. Improvement of digestive health and reduction in proteobacterial populations in the gut microbiota of cystic fibrosis patients using a Lactobacillus reuteri probiotic preparation: a double blind prospective study. J Cyst Fibros 2014;13:716-22.

101. Odamaki T, Sugahara H, Yonezawa S, Yaeshima T, Iwatsuki $\mathrm{K}$, Tanabe $\mathrm{S}$, et al. Effect of the oral intake of yogurt containing Bifidobacterium longum BB536 on the cell numbers of enterotoxigenic Bacteroides fragilis in microbiota. Anaerobe 2012;18:14-8.

102. Marteau P. Evidence of probiotic strain specificity makes extrapolation of results impossible from a strain to another, even from the same species [Internet]. Ann Gastroentol Hepatol: c2011 [cited 2021 Apr 12]. Available from: http://gastromasterclass2014. s3.amazonaws.com/Session1/Marteau+-+Evidence+of +probiotic+strain+specificity.pdf.

103. Swanson KS, Gibson GR, Hutkins R, Reimer RA, Reid $G$, Verbeke $K$, et al. The International scientific association for probiotics and prebiotics (ISAPP) consensus statement on the definition and scope of synbiotics. Nat Rev Gastroenterol Hepatol 2020;17:687-701.

104. Hadi A, Alizadeh K, Hajianfar H, Mohammadi H, Miraghajani M. Efficacy of synbiotic supplementation in obesity treatment: a systematic review and meta-analysis of clinical trials. Crit Rev Food Sci Nutr 2020;60:584-96.

105. Ahmadi S, Jamilian M, Tajabadi-Ebrahimi M, Jafari P, Asemi Z. The effects of synbiotic supplementation on markers of insulin metabolism and lipid profiles in gestational diabetes: a randomised, double-blind, placebo-controlled trial. Br J Nutr 2016;116:1394-401.

106. Zare Javid $A$, Aminzadeh $M$, Haghighi-Zadeh $M H$, Jamalvandi $M$. The effects of synbiotic supplementation on glycemic status, lipid profile, and biomarkers of 


\section{Obstetrics \& Gynecology Science}

Vol. 65 , No. 1, 2022

oxidative stress in type 1 diabetic patients. A placebocontrolled, double-blind, randomized clinical trial. Diabetes Metab Syndr Obes 2020;13:607-17.

107. Tajabadi-Ebrahimi M, Sharifi N, Farrokhian A, Raygan F, Karamali $F$, Razzaghi $R$, et al. A randomized controlled clinical trial investigating the effect of synbiotic administration on markers of insulin metabolism and lipid profiles in overweight type 2 diabetic patients with coronary heart disease. Exp Clin Endocrinol Diabetes 2017;125:21-7.

108. Rodgers RJ, Avery JC, Moore VM, Davies MJ, Azziz R, Stener-Victorin $\mathrm{E}$, et al. Complex diseases and co-morbidities: polycystic ovary syndrome and type 2 diabetes mellitus. Endocr Connect 2019;8:R71-5.

109. Zore T, Joshi NV, Lizneva D, Azziz R. Polycystic ovarian syndrome: long-term health consequences. Semin Reprod Med 2017;35:271-81.

110. Wu J, Yao XY, Shi RX, Liu SF, Wang XY. A potential link between polycystic ovary syndrome and non-alcoholic fatty liver disease: an update meta-analysis. Reprod
Health 2018;15:77.

111. Esmaeilinezhad Z, Babajafari S, Sohrabi Z, Eskandari $\mathrm{MH}$, Amooee S, Barati-Boldaji R. Effect of synbiotic pomegranate juice on glycemic, sex hormone profile and anthropometric indices in PCOS: a randomized, triple blind, controlled trial. Nutr Metab Cardiovasc Dis 2019;29:201-8.

112. Darvishi S, Rafraf M, Asghari-Jafarabadi M, Farzadi L. Synbiotic supplementation improves metabolic factors and obesity values in women with polycystic ovary syndrome independent of affecting apelin levels: a randomized double-blind placebo - controlled clinical trial. Int J Fertil Steril 2021;15:51-9.

113. Karimi E, Moini A, Yaseri M, Shirzad N, Sepidarkish $M$, Hossein-Boroujerdi $M$, et al. Effects of synbiotic supplementation on metabolic parameters and apelin in women with polycystic ovary syndrome: a randomised double-blind placebo-controlled trial. $\mathrm{Br} J$ Nutr 2018;119:398-406. 